\title{
RESEARCHARTICLE
}

\section{The Effect of Heel Lifts on Postural Stability in Individuals with Parkinson's Disease}

\author{
Jennifer D. Hastings*, Connor Brown, McKenna McNabb, and Cassie Repasky \\ *School of Physical Therapy, University of Puget Sound, USA
}

\begin{abstract}
Our purpose was to investigate if accommodating plantar flexion contractures will improve postural alignment, patient perceived stability, and balance in individuals with Parkinson's disease (PD), and the correlation of plantarflexion (PF) contractures and the Falls Efficacy scale (FES). This was a single session pretest posttest study with subject as their own control. We recruited a convenience sample of 32 participants with PD who could independently ambulate 20 feet without assistive devices. The Institutional Review Board approved the study, and informed consent was obtained from all participants prior to their participation. The outcome measures included: sagittal plane posture photo, verbal numeric rating of perceived stability, 1-minute recording of double leg stance on an interface pressure map, functional reach test (FRT) and the Falls Efficacy Scale (FES). The intervention was the use of bilateral Adjust-a-Lift heel lifts worn inside the participants' shoe. Assigned heel lift thickness was based on PF contracture severity, determined via passive talocrural dorsiflexion measurement. Photographic posture analysis was completed with free image measurement software. Sway measurements were taken of center of gravity tracing with Tekscan software. Statistical analysis included Paired t-tests for outcome measures and Pearson product correlation between the FES and PF contracture data. We found significant changes $(p<.05)$ in increase in height (mean 1.00 $\mathrm{cm}$ ), an anterior translation of the pelvis (mean $2.3 \mathrm{~cm}$ ), a more upright trunk angle (mean 1.37 degrees) and a more upright head angle (mean 2.61 degrees).Perceived stability significantly increased (mean 0.75) and functional reach significantly decreased (mean $2.22 \mathrm{~cm})$. Significant correlations $(r=0.36-0.50)$ were found between the degree of PF contracture and the total scale score as well as reported fear of falling for questions that required walking on the FES. This study showed accommodating PF contractures with heel lift intervention significantly improved postural alignment and perceived stability, but decreased participants' functional reach.
\end{abstract}

\section{Keywords}

Parkinson's disease, posture, contracture, plantarflexion

\section{Introduction}

Parkinson's Disease (PD) is a progressive, neurodegenerative condition that reduces the basal ganglia's dopamine production in the substantia nigra. ${ }^{1}$ Dopamine is an important neurotransmitter for multiple body functions including movement proficiency. Due to the lack of dopamine, people with PD commonly present with four primary motor symptoms: resting tremors, bradykinesia, rigidity, and postural instability. ${ }^{2,3}$ While extensive research is aimed at treating tremors, bradykinesia, and rigidity, little research has analyzed the relationship between posture and stability.

Stability, or balance, is an essential component to preventing falls. Fall prevention is a critical topic in the PD population as falling is responsible for $30 \%$ of PD hospital admissions. ${ }^{4}$ A recent study estimated that people with PD are 2-9 times more likely to fall, $50 \%$ of the PD population report falls, and $30 \%$ of those falls result in injury. ${ }^{2}$ Furthermore, postural instability has been identified as an independent predictor of falls and a third of people with PD develop 
postural instability within 2 years of diagnosis. ${ }^{2}$ Pharmacological research suggests that poor balance in this population is due to malfunctioning afferent sensory integration and decreased efferent neuromuscular signals in the central nervous system (CNS) ${ }^{5,6}$ However there is also evidence that balance can worsen with these pharmacological interventions, suggesting there are other contributors to fall risk in this population. ${ }^{2,7}$

Upright posture is maintained by controlling the center of pressure relative to the vertical position of the center of gravity (COG), therefore, an optimal postural alignment is the foundation of energy efficiency, balance, and fall prevention. Anatomically optimal posture is most effective at withstanding constant gravitational forces with the least amount of energy, it is described as the COG aligned anterior to the talocrural joint, anterior to the knee, slightly posterior to the hip, and through the lumbar and cervical vertebral bodies and the external auditory meatus (i.e. the plumb line). ${ }^{8}$ This preferred alignment uses bony and ligamentous architecture for support and minimizes muscular effort for stability so phasic and tonic muscles can be recruited appropriately. This posture also maximizes available room for movement in each direction, which is essential for preventing loss of balance (balance being defined as maintaining COG inside the base of support (BOS) while reacting to external perturbations).

People with PD adapt a standing alignment often referred to as a "stooped posture", characterized by flexed hips, kyphotic spine, forward shoulders, forward head, and downward head tilt. ${ }^{5,9}$ For this study the authors are most interested in the hip flexion aspect of this posture. The hip joint is between the pelvis and femur and in the upright position with hip flexion there are three potential compensations: 1) flexion of the knee, 2) increased lumbar extension (lordosis), and 3) posterior translation of the pelvis with concomitant posterior translation of the tibial and plantarflexion. This population has a high prevalence of impaired standing and dynamic balance, decreased muscle strength, slower gait, and slower anticipatory and reactionary balance. ${ }^{6}$ We propose that plantarflexion contractures are a biomechanical contributor to imbalance and the "stooped posture" adaptation.

Contractures develop when muscles are maintained at shortened lengths for long periods of time. ${ }^{10}$ Shortened stride length, shuffling feet, early heel rise and reduced push off at terminal stance (all common in PD) significantly reduce the excursion of the plantarflexor muscles and may promote the development of contractures. Plantarflexion contractures effectively shorten the available dorsiflexion range of motion (ROM) which causes posterior translation of the pelvis in the sagittal plane, and creates a flexion moment at the hips (anterior pelvic rotation) or trunk to maintain the COG inside the BOS. Although no research has identified the prevalence of PF contractures in the PD population, various studies have looked at foot and ankle characteristics in the elderly population, and found decreased dorsiflexion ROM significantly correlated with impaired balance and functional ability in older adults. ${ }^{11,12}$

Evidence indicates that stretching a contracture does not significantly improve ROM. ${ }^{8}$ One study by Bartonek et al. was able to optimize standing posture alignment, in children with Cerebral Palsy (CP), with the use of heel lifts to accommodate PF. ${ }^{13}$ Although CP can have a wide variety of presentations; the progressive postural changes seen in PD are predictable, theoretically making an effective intervention appropriate for many people with the diagnosis. In theory, accommodating plantarflexion contractures will change the upright alignment by reducing the posterior translation of the tibial and allowing more neutral position of joints above.

The aim of this study was to determine if accommodating plantar flexion contractures with a heel lift would improve postural alignment and stability.

\section{Materials and Methods}

We selected the outcome measures to capture different aspects of balance, stability, and postural alignment. The sagittal photo captured participants' postural alignment, the Tekscan interface pressure map captured their COG tracing, the verbal numeric rating scale of 0-10 captured participants' perceived stability, and the forward reach test (FRT) captured an aspect of dynamic balance. Literature suggests the FRT is a valid assessment of balance for people with PD. ${ }^{14,15}$ Additionally, the Falls Efficacy Scale (FES) was selected because it has been found to be a reliable and valid measure in a PD sample that assesses an individual's perceived ability and fear concerning activities of daily living (ADL) performance and 
community participation. ${ }^{16,17}$ Nilsson reported test-retest reliability of FES at 0.87 with standard error of measurement at 12.3, no minimal detectable difference has been established for the population. ${ }^{16}$ We wanted to see if low levels of activity and community participation (FES scores) correlated with degrees of PF contracture severity.

\section{Participants}

32 Participants were recruited via flyers and announcements at community support groups for Parkinson's Disease in the Seattle-Tacoma area and by word-of-mouth referral. Participants were included if they were able to ambulate $20 \mathrm{ft}$. with or without an assistive device, and excluded if they were unable to understand English or had a pre-existing medical condition that would impact their postural stability. The Institutional Review Board of University of Puget Sound approved the study, and informed consent was obtained from all participants prior to their participation. Participants spent 1 hour at the University of Puget Sound PT clinic for the single day of data collection.

Figure 1.Study Layout

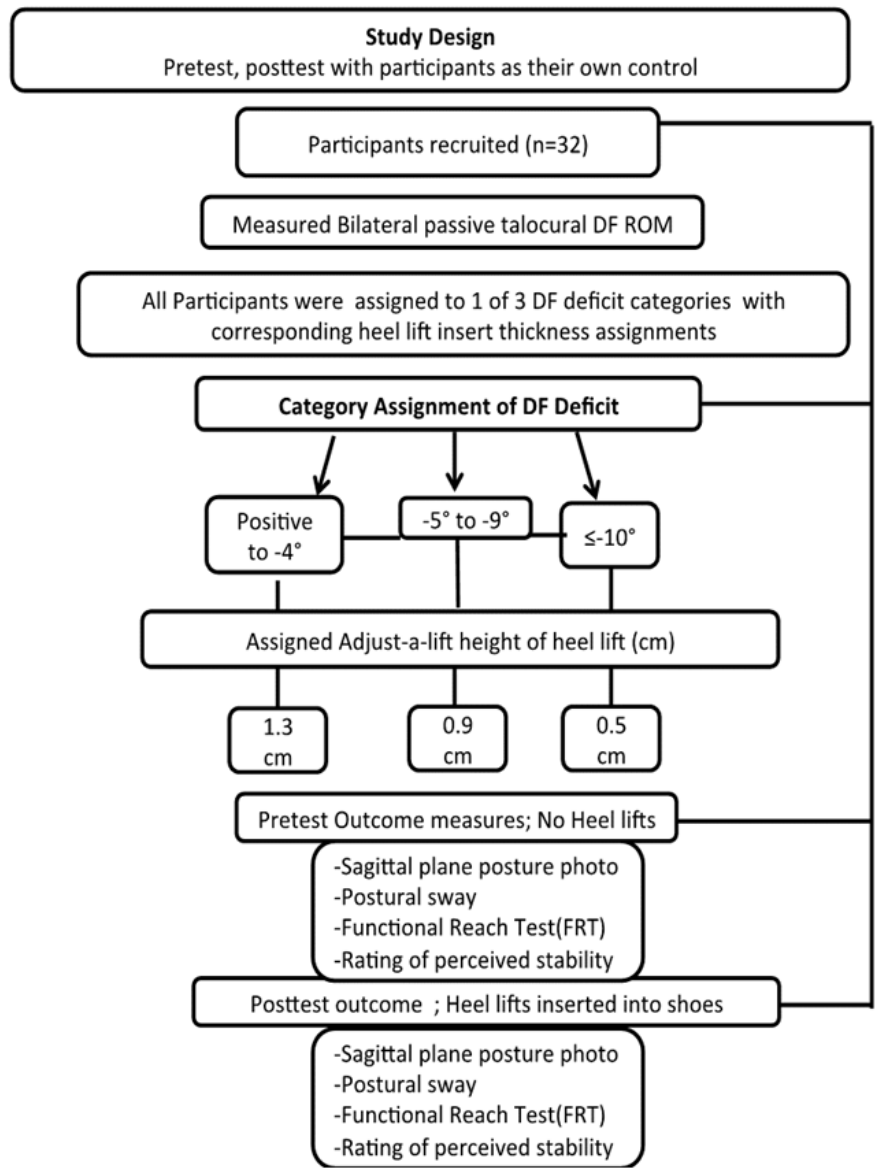

See Figure 1 for the study flowchart. Our study was a single session pretest post test design with subjects as their own control. After consent, participants completed the FES. The FES is a 16-question scale assessing the latent construct of fear of falling. Per the outcome measure instructions, the researcher explained that the questions were about "how concerned you are about falling" and the participant was instructed to "think about how they usually do the activity". Answer options are: Not at all concerned, somewhat concerned, fairly concerned, and very concerned and each answer corresponds to 1-4 for scoring; the higher score representing a higher level of concern. Next passive DF range of motion was measured, then each participant was then taken through the first round of 4 outcome measure stations where data was collected on their sagittal posture, perceived stability, standing postural sway, and functional reach. Following the first round (baseline), a heel lift was inserted into their shoes, and they repeated each station.

\section{Talocrural DF ROM and Heel Lift Assignment}

Passive talocrural DF ROM was assessed at baseline and was used to determine the height of the heel lift placed into participants' shoes. The measurement was conducted with the 
participants in supine, knees extended, and in subtalar neutral. Using a plastic goniometer, the fulcrum was the lateral body of calcaneus, the stationary arm was aligned with the fibular shaft and the moving arm was aligned parallel to the body of the calcaneus. All dorsiflexion measurements were done by the same licensed physical therapist. Diamond et al found that passive DF measurement by physical therapists with a standard goniometer was reliable, reporting intraclass correlation coefficients of .89 for right $(R)$ ankle and .96 for the left $(L)$ ankle, with a standard error of measurement of 1-degree $L$ and 3-degrees R.18 More recently, Konor et al found nearly the same ICC of .85 R and .96 L for weight bearing measurement of DF with a standard goniometer. ${ }^{19}$

The side with the more severe plantar flexion contracture (the largest DF ROM deficit) determined which size heel lifts used bilaterally in the participants' shoes. The Warwick Enterprises Adjust-a-Lift heel lifts were used for this study. The accommodating heel lift size thickness included $0.5 \mathrm{~cm}, 0.9 \mathrm{~cm}$, and $1.3 \mathrm{~cm}$. See Table 1 for DF range and heel lift assignment.

Table 1. Dorsiflexion Range of Motion and the Intervention Group Categorization

\begin{tabular}{|c|c|c|c|}
\hline $\begin{array}{c}\text { Dorsiflexion } \\
\text { range (degrees) }\end{array}$ & $\begin{array}{c}\text { Mean DF } \\
\text { degrees (SD) }\end{array}$ & $\begin{array}{c}\text { Number of } \\
\text { participants }\end{array}$ & $\begin{array}{c}\text { Heel lift } \\
\text { size (cm) }\end{array}$ \\
\hline Positive to -4 & $2.4(3.99)$ & 24 & 0.5 \\
\hline-5 to -9 & $-6.5(1.97)$ & 6 & 0.9 \\
\hline$\leq-10$ & $-12(2.82)$ & 2 & 1.3 \\
\hline
\end{tabular}

\section{Posture}

A photo was taken of each participant in the sagittal plane before and after heel lift insertion, for software analysis. Participants were instructed to place their toes against a 10 " $\times 5$ " box fixed to the ground, and to stand comfortably while looking at a fixed picture on the wall in front of them. A standardized camera position was used. See Figure 2 for an example of the standardized picture taken.

Figure 2. Posture measurements example

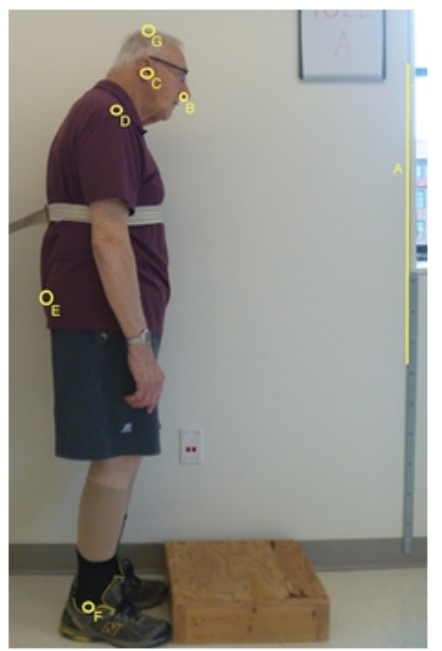

Points are for display purposes only. Actual points of measurement were taken with far more precision

The posture photographs were analyzed using free image measurement software called "Image-J."The measurements were standardized with the same 1-meter yardstick. Image-J software sets each picture on a pixel grid overlaying a graph: $\mathrm{x}$-axis is horizontal and $\mathrm{y}$-axis is vertical. Linear horizontal measurements $(\mathrm{cm})$ were taken from point $A$ (reference point to known vertical) to Points B (base of nares), C (external acoustic meatus), D (acromion), and E (posterior pelvis). The angular measurement (degrees) for the trunk was taken as the angle formed between two straight lines: point C-Point E and Point E-Horizontal anteriorly. Then angular measurement for head tilt was measured as the angle formed between point B-point $C$ and Point C- vertical inferiorly. The overall height was measured as the linear distance between point $F$ (lateral malleolus) and Point $G$ (top of the head).See Figure 2 for measurements taken. A single researcher did all of the photographic measurement and test- 
retest measurements were consistently within $1 \mathrm{~cm}$ and $1 / 10$ of a degree.

\section{Perceived Stability}

During both rounds at the posture photo station participants were asked to rate their perceived stability on a verbal numeric scale with anchors of 0 (least stable) to 10 (most stable). This outcome measure is similar to a common clinical tool use for assessment of pain intensity. Visual Analog Scales and Numeric Pain Rating scales with defined anchors have been found to be reliable and valid, ${ }^{20}$ and the verbal numeric rating scale has been shown to have good agreement with a written scale. ${ }^{21}$ There is no gold standard for perceived stability.

\section{Postural Sway}

Participants' postural sway was assessed via a COG tracing from an interface pressure mapping movie. A Tekscan Body Pressure Mapping System sensor mat was used. Participants were instructed to stand comfortably in the middle of the sensor mat that was on the floor, look straight ahead, and refrain from excessive movement. The Tekscan pressure mapping movie recorded 400 frames over 60 seconds and was saved for future analysis. Tekscan CONFOR Mat Clinical 7.60 software was used to measure the greatest anterior posterior distance of the center of gravity tracing and then the greatest width perpendicular to the first measure.

\section{Functional Reach Test}

Instructions for the FRT outcome measure were followed by those provided on rehabmeasures.org. For both pre and post intervention rounds, the participants' functional reach was measured to the 10th of an inch, with a yardstick set at the level of the participants' acromion. Participants were instructed to hold their left arm up with their hand in a fist to the level of the yardstick. Next, participants were asked to reach as far as possible while keeping their fist level with the yardstick, and without moving their feet (i.e. taking a step forward or losing balance). Reaches were repeated three times; with a 10-15 second break in between trials. The first of the three trials was considered as practice; the last two reaches were averaged for analysis.

Statistical analysis was completed using SPSS version 23 software. The postural photo measurements, TekScan pressure mapping measurements, perceived stability numeric scores, and the functional reach test measurements were analyzed using a paired-t test comparing round 1 (baseline) to round 2 (lift). Data from the FES was correlated to the DF ROM deficits. For simplicity and consistency, the functional reach difference was converted from inches to centimeters.

\section{Results}

The study sample comprised thirty-two adults ( $n=32)$ with PD (19 male and 13 female) with an average age of 70 years (range of 43-83). The average time since diagnosis was 5.4 years, and ranged from 1 month to 17 years prior to the study. Six of our participants reported freezing episodes and on average the participants reported less than one fall in the last month (0-4).

The mean of the participants' talocrural DF ROM was 0.17 degrees, and ranged between negative 10 and 16 degrees. Most participants had asymmetry in their DF ROM with a mean of 4.6 degrees difference between feet. No participant had a normal dorsiflexion range (described by the American Medical Association as 20 degrees past neutral) and a total of 8 participants had ankle DF ROM $\geq 5$ degrees. 
Table 2. Results of Paired t Test for Outcome Measures

\begin{tabular}{|c|c|c|c|c|}
\hline Outcome Measure & $\begin{array}{l}\text { Mean Change } \\
\text { With Lifts }\end{array}$ & Lower $95 \% \mathrm{Cl}$ & Upper $95 \%$ Cl & p value \\
\hline Posterior pelvis change $¥$ & $2.3 \mathrm{~cm}$ anterior & $1.4 \mathrm{~cm}$ & $3.1 \mathrm{~cm}$ & $.000^{*}$ \\
\hline Trunk angle from horizontal $¥$ & $\begin{array}{c}1.37 \text { degrees } \\
\text { bigger }\end{array}$ & $\begin{array}{c}47 \text { degrees } \\
\text { bigger }\end{array}$ & $\begin{array}{l}2.55 \text { degrees } \\
\text { bigger }\end{array}$ & $.004^{*}$ \\
\hline Head tilt angle from vertical $¥$ & $\begin{array}{l}2.61 \text { degrees } \\
\text { bigger }\end{array}$ & $\begin{array}{c}.30 \text { degrees } \\
\text { bigger }\end{array}$ & $\begin{array}{l}4.93 \text { degrees } \\
\text { bigger }\end{array}$ & $.028^{*}$ \\
\hline Overall height $¥$ & $1.0 \mathrm{~cm}$ taller & $0.3 \mathrm{~cm}$ taller & $1.7 \mathrm{~cm}$ taller & $.007^{*}$ \\
\hline Functional reach & $2.23 \mathrm{~cm}$ shorter & $0.90 \mathrm{~cm}$ shorter & $3.55 \mathrm{~cm}$ shorter & $.002^{*}$ \\
\hline $\begin{array}{c}\text { Perceived stability } \\
\text { Verbal Numeric Scale Score }\end{array}$ & .75 & .270 & 1.20 & $.003^{*}$ \\
\hline $\begin{array}{l}\text { COG tracing } \\
\text { AP distance }\end{array}$ & $.16 \mathrm{~cm}$ & $-.33 \mathrm{~cm}$ & $.65 \mathrm{~cm}$ & .511 \\
\hline $\begin{array}{c}\text { COG tracing } \\
\text { Horizontal }\end{array}$ & -.115 & -.694 & .463 & .687 \\
\hline
\end{tabular}

$¥$ indicates measurements from photo analysis. * indicates significant at $\alpha=0.05$

Table 3. Relationship between severity of PF contracture and Falls Efficacy Scale

\begin{tabular}{|l|c|c|}
\hline Pearson Product Correlation & $r$ & $p$ \\
\hline Total Scale Score & .41 & .020 \\
\hline \#8: Walking around the neighborhood & .46 & .012 \\
\hline \#14: Walking on an uneven surface & .36 & .046 \\
\hline \#15 Walking up or down a slope & .42 & .018 \\
\hline \#16 Going out to a social event & .40 & .030 \\
\hline
\end{tabular}

Figure 3. Example for significant findings for change in trunk and head angles at baseline (left), and with the heel lifts in (right)

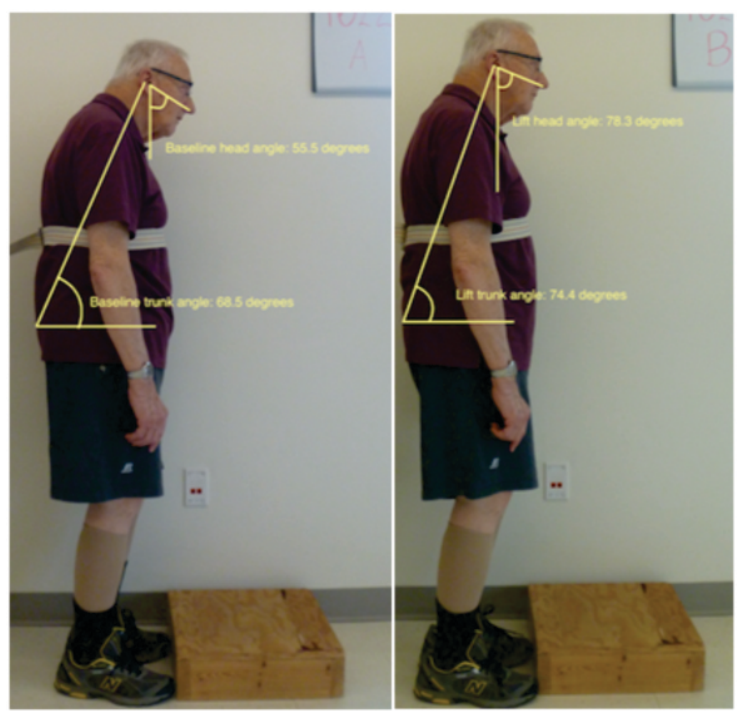


Figure 4. Example of significant findings for change in pelvis position and overall height comparing baseline (left) and heel lift (right)

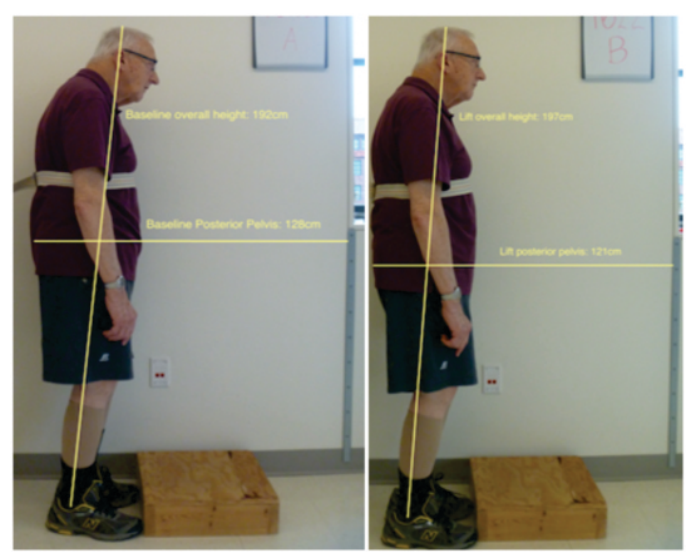

Table 2 describes the significant changes seen with the heel lifts in shoes. On average, when wearing the shoe heel lifts, the participants' pelvis moved anteriorly, their trunk and head shifted to be more upright, they were taller, and their perceived stability improved. Figures 3 and 4 illustrate the changes noted in one participant's postural alignment photos. This participant increased his head angle by 2.8 degrees and trunk angle by 5.9 degrees with his pelvis shifting $7 \mathrm{~cm}$ anteriorly and his height increasing $5 \mathrm{~cm}$. No significant changes were found in the postural sway data and a significant decline in functional reach ability was observed.

As seen in table 3 there was a significant correlation between the measured DF ROM deficit and the FES total scale score as well as questions $8,14,15$, and 16, of the FES. These four questions address an individual's fear of falling when walking around the neighborhood, walking on uneven terrain, walking on a slope, or when participating in a social event.

\section{Discussion}

The findings of this study provide preliminary evidence that heel lifts immediately move posture closer to anatomical plumbline, and improve perceived stability in people with PD, but decrease their reach excursion in the FRT. Our sample was too small to analyze the influence of age or disease severity on the effectiveness of the intervention. However, as seen in figures 3 and 4 we did see improvements in individuals with advanced age and more severity. Surprising to us was that we also so improvements when there was minimal DF deficit.

In this study, we were unable to find any changes in postural sway. This may have been a limitation of our equipment as we did not have access to a specific postural sway system and used an interface pressure sensor for body pressure mapping, software not specific to sway analysis, and had the participants wearing shoes. Individuals who had uneven weight bearing without heel lifts did tend to map more symmetrically with the heel lifts. However, there was no significant difference in the size of the center of gravity tracing with or without heel lifts.

As observed in the posture photos, the participants' pelvis translated anteriorly in the sagittal plane which opened their trunk angle and caused them to stand in an enhanced upright alignment closer to anatomically optimal posture. Theoretically, this decreases the muscular demands of the posterior extensor chain muscles as the skeletal system is in a better position to support body weight.

When combined with bradykinesia and rigidity, decreased ROM can significantly hinder reactive strategies to correct a loss of balance. Lack of necessary ROM decreases the effectiveness of hip and ankle balance strategies, impeding the ability to regain balance following an external perturbation. ${ }^{22}$ The postural improvements seen in our participants create larger ROM available for knee, hip, and lumbar flexion, which is essential for balance reactions. This alignment change most likely explains the improvements seen in perceived stability, for we effectively increased the available excursion for functional balance adjustments. It should be noted that none of our participants moved into a perfect upright alignment. The remaining postural deficits likely reflect some musculoskeletal shortening or lack of free motion at other proximal joints. This theory suggests that heel lifts may be a useful treatment to postural alignment; however, they should be considered an adjunct treatment to 
joint mobilization and muscle lengthening techniques.

Interestingly, participants' forward reach excursion significantly decreased with heel lifts. As the FRT is used frequently in literature as a balance measure, we initially hypothesized that the intervention to improve upright posture would result in better balance and a longer reach. By accommodating the PF contractures our intervention made the primary balance compensation, of rearward pelvic counterbalance, more difficult. Upon reflection, we argue that FRT is not an appropriate marker for upright postural alignment and postural stability. The instructions for FRT do not allow the feet to move and therefore the task requires redistribution of the body over the feet to counterbalance for forward reach, it is therefore a good test of available dynamic realignment. Therapeutically, one would not encourage this maneuver for an activity of daily living (truly "functional" reaching) but rather encourage the more natural stepping a foot forward to increase the base of support and facilitate reach. Future studies should look at how the use of heel lifts might impact the ease of stepping.

Significant correlation was found between the FES and PF contracture severity. This is a critical finding because it suggests both PF contractures and the FES can be utilized to determine the degree of ADL and participation deficits. Additionally, the FES subscales that correlated with the degree of PF contractures all involved ambulation, suggesting that severity of PF contractures affects difficulty and balance deficits during ambulatory activities.

Our study suggests the heel lifts improved participants' perceived stability, which is another way of ascertaining how comfortable they feel in preventing falls. Many studies have found that reducing one's fear of falling decreases risk of future falls. ${ }^{23,24}$ Thus, we suggest that accommodating PF contractures with a heel lift would also decrease fall risk in the PD population. Additionally, we predict that in addition to postural alignment, dynamic balance should also improve with PF accommodation because of the increased excursion available for balance corrections and reactions. For instance, several studies that have shown that heel lifts can improve alignment and balance in children with CP and older adults. ${ }^{12,25}$ Furthermore, according to our FES and PF correlation data, the PD population has increased difficulty with ADL's involving ambulation (i.e. dynamic balance). To support these theories, further research needs to be conducted, aiming to analyze the effects heel lifts have on dynamic balance in the PD population.

\section{Limitations}

The two primary limitations to our study are the single session design and static (instead of dynamic) posture in single plane, which might not provide a comprehensive view of the effects of our intervention. We cannot know if the heel lift's immediate effects we observed in this study would be maintained over time, nor if there are any negative consequences of full time heel lift intervention. This indicates a need for a long-term follow-up to determine if dynamic balance improves with the use of heel lifts over time, or if training dynamic balance with the heel lifts is needed to improve this aspect of their balance. Another limitation of the study is that the sensitivity of the Tekscan equipment did not detect any significant differences in the participants' COG. Also, this study suggests that the use of FRT should be reconsidered as a stability measure. Therefore, future research needs to be conducted using more sensitive equipment to detect COG shifts, applying another valid test for PD to look at dynamic balance, and including a long-term follow up.

\section{Conclusion}

This study showed accommodating PF contractures with heel lifts provided significant immediate improvement in postural alignment and perceived stability, but decreased participants' functional reach test distance. There is a need to perform a long-term follow-up to determine if these findings can be sustained. 


\section{References}

1. Goodman CC, Fuller KS. Pathology: Implications for the Physical Therapist. 4th ed. St. Louis: Elsevier Saunders.

2. Klamroth S, Steib S, Devan S, Pfeifer K. Effects of Exercise Therapy on Postural Instability in Parkinson Disease: A Meta-analysis. J Neurol Phys Ther JNPT. 2016;40(1):3-14. doi:10. 1097/NPT.0000000000000117.

3. Gepshtein S, Li X, Snider J, et al. Dopamine function and the efficiency of human movement. J Cogn Neurosci. 2014;26(3):645-657. doi:10.1162/jocn_a_00503.

4. Oguh O, Videnovic A. Inpatient Management of Parkinson Disease. The Neurohospitalist. 2012;2(1):28-35. doi:10.1177/1941874411427734.

5. Rogers ME, Page P, Takeshima N. Balance Training for the older athlete. Int J Sports Phys Ther. 2013;8(4):517-530.

6. Jankovic J. Parkinson's disease: clinical features and diagnosis. J Neurol Neurosurg Psychiatry. 2008;79(4):368-376. doi:10.1136/jnnp.2007.131045.

7. Schoneburg B, Mancini M, Horak F, Nutt JG. Framework for understanding balance dysfunction in Parkinson's disease. Mov Disord Off J Mov Disord Soc. 2013;28(11):1474-1482. doi:10. $1002 /$ mds. 25613.

8. Colby K. Therapeutic Exercise: Foundations and Techniques. 6th ed. Philadelphia: FA Davis; 2012.

9. Forsyth AL, Paul SS, Allen NE, et al. Flexed Truncal Posture in Parkinson Disease: Measurement Reliability and Relationship With Physical and Cognitive Impairments, Mobility, and Balance. J Neurol Phys Ther JNPT. 2017;41(2):107-113. doi:10.1097 /NPT. 00000 00000000171.

10. Harvey LA, Herbert RD. Muscle stretching for treatment and prevention of contracture in people with spinal cord injury. Spinal Cord. 2002;40(1):1-9. doi:10.1038/sj.sc.3101241.

11. Liao YY, Yang YR, Wu YR, Wang RY. Factors Influencing Obstacle Crossing Performance in Patients with Parkinson's Disease. PLoS ONE. 2014;9(1). doi:10.1371/journal.pone.0084245.

12. Menz HB, Morris ME, Lord SR. Foot and ankle characteristics associated with impaired balance and functional ability in older people. J Gerontol A Biol Sci Med Sci. 2005; 60(12):1546-1552.

13. Bartonek A, Lidbeck CM, Pettersson R, Weidenhielm EB, Eriksson M, Gutierrez-Farewik E. Influence of heel lifts during standing in children with motor disorders. Gait Posture. 2011;34(3):426-431. doi:10.1016/j.gaitpost.2011.06.015.

14. Behrman AL, Light KE, Flynn SM, Thigpen MT. Is the functional reach test useful for identifying falls risk among individuals with Parkinson's disease? Arch Phys Med Rehabil. 2002; 83(4):538-542.

15. http://www.rehabmeasures.org/default.aspx. The Rehabilitation Measures Database. http://www.rehabmeasures.org/default.aspx. Accessed October 22, 2016.

16. Nilsson MH, Drake AM, Hagell P. Assessment of fall-related self-efficacy and activity avoidance in people with Parkinson's disease. BMC Geriatr. 2010;10:78. doi:10.1186/1471-2318-10-78.

17. Jonasson SB, Ullén S, Iwarsson S, Lexell J, Nilsson MH. Concerns About Falling in Parkinson's Disease: Associations with Disabilities and Personal and Environmental Factors. J Park Dis. 2015;5(2):341-349. doi:10.3233/JPD-140524.

18. Diamond JE, Mueller MJ, Delitto A, Sinacore DR., Reliability of a diabetic foot evaluation.Phys Ther. 1989 Oct;69(10):797-802. Erratum in: Phys Ther. 1989;69(11): 994.

19. Konor MM, Morton S, Eckerson JM, Grindstaff TL, Reliability of Three Measures of Ankle Dorsiflexion Range of Motion. The Int J Sports Phy Ther. 2012;7(3): 279.

20. Kahl C, Cleland JA. Visual analogue scale, numeric pain rating scale and the McGill pain Questionnaire: an overview of psychometric properties, Phy Ther Rev. 2005;10(2): 123-128. DOI: $10.1179 / 108331905 \times 55776$

21. Paice JH, Cohen FI, Validity of a Verbally Administered Numeric Rating Scale to Measure Cancer Pain Intensity. CancerNurs. 1997'20(2): 88-93. 
22. Chiacchiero M, Dresely B, Silva U, DeLosReyes R, Vorik B. The Relationship Between Range of Movement, Flexibility, and Balance in the Elderly. Topics Geriatric Rehab. 210;26(2): $147-154$.

23. Mak MKY, Pang MYC. Fear of falling is independently associated with recurrent falls in patients with Parkinson's disease: a 1-year prospective study. J Neurol. 2009;256(10):1689-1695. doi:10.1007/s00415-009-5184-5.

24. Lindholm B, Hagell P, Hansson O, Nilsson MH. Factors associated with fear of falling in people with Parkinson's disease. BMC Neurol. 2014;14:19. doi:10.1186/1471-2377-14-19.

25. Gross MT, Mercer VS, Lin FC. Effects of foot orthoses on balance in older adults. J Orthop Sports Phys Ther. 2012;42(7):649-657. doi:10.2519/jospt.2012.3944. 